\title{
Hexavalent Chromium in Waters for Human Consumption and Irrigation en the Guano Canton
}

\section{Cromo Vi en Aguas de Consumo Humano y de Riego en el Cantón Guano}

VII International Congress of

Science, Technology,

Entrepreneurship and

Innovation (SECTEI 2020)

Corresponding Author:

G Castillo-López

maria.vinueza@espoch.edu.ec

Published: 26 August 2021

Production and Hosting by

Knowledge E

(c) G Castillo-López et al. This article is distributed under the terms of the Creative Commons Attribution License, which permits unrestricted use and redistribution provided that the original author and source are credited.

\author{
G Castillo-López ${ }^{1}$, P Salas-Cisneros ${ }^{1}$, MA Logroño-Veloz ${ }^{2}$, and MF Vinueza- \\ Veloz ${ }^{3}$ \\ ${ }^{1}$ Hospital General de Ibarra, Ibarra, Imbabura, Ecuador \\ ${ }^{2}$ Carrera de Nutrición y Dietética, Facultad de Salud Pública, Escuela Superior Politécnica de \\ Chimborazo, Riobamba, Chimborazo, Ecuador \\ ${ }^{3}$ Carrera de Medicina, Facultad de Salud Pública, Escuela Superior Politécnica de Chimborazo, \\ Riobamba, Chimborazo, Ecuador
}

\section{Abstract}

Introduction: Considering the geographical location and industrial activities of the county Guano, which lies on an aquifer that supply water for both consumption and crop irrigation, it is necessary to examine the levels of chromium $(\mathrm{Cr})$ in its watershed and tributaries. Objective: To evaluate the amount of $\mathrm{Cr}$ existing in the surface waters of the Guano river basin and aquifer Methods: Several samples of the river and aquifer were taken and the amount of $\mathrm{Cr}$ present was measured. Using Z-test, $\mathrm{Cr}$ levels found in the water were compared with those recommended as normal by the World Health Organization (WHO), national standards of the Texto Unificado de Legislación Secundaria de Medio Ambiente (TULSMA) and the Servicio Ecuatoriano de Normalización 1108 (INEN 1108). Results: Relatively high $\mathrm{Cr}$ values were found when compared with those established by the WHO. However, they were normal when compared with TULSMA and INEN 1108. Conclusion: The levels of $\mathrm{Cr}$ found in the Guano river and the aquifer were higher than those recommended by the WHO. It is recommended to replicate our findings in order to corroborate the results and to take appropriate measures in order to reduce water contamination.

Keywords: chromium, water, Guano, Ecuador

\section{Resumen}

Introducción: Considerando la ubicación geográfica y actividades industriales del cantón Guano el cual yace sobre un acuífero que sustenta las necesidades de agua tanto para consumo y riego de la población es necesario examinar los niveles de Cromo $(\mathrm{Cr})$ en su vertiente como sus afluentes. Objetivo: Evaluar la cantidad de $\mathrm{Cr}$ existente en las aguas superficiales de la cuenca del río Guano y en pequeños ojos de agua provenientes del acuífero. Métodos: Se tomó varias muestras de agua del río y ojos de agua y se midió la cantidad de $\mathrm{Cr}$ presente. Por medio de la prueba $\mathrm{Z}$ se comparó los niveles de $\mathrm{Cr}$ con los recomendados como normales por la Organización Mundial de la Salud (OMS), normas nacionales del Texto Unificado de Legislación Secundaria de Medio Ambiente (TULSMA) y Servicio Ecuatoriano de Normalización 1108 (INEN 1108). Resultados: Se encontró valores relativamente altos de $\mathrm{Cr}$ al compararlos con los establecidos por la OMS. Sin embargo, estos se encontraron dentro de 
rangos normales al compararlos con TULSMA e INEN 1108. Conclusiones: Los niveles de $\mathrm{Cr}$ encontrados en el río Guano y ojos de agua del acuífero son superiores a los recomendados por la OMS. Se recomienda replicar los hallazgos para corroborar los resultados y tomar las medidas adecuadas para reducir la contaminación del agua.

Palabras Clave: cromo, agua, Guano, Ecuador

\section{Introducción}

El cromo es un metal necesario para la vida y de uso común en la industria. El $\mathrm{Cr}$ está presente en formas estables e inestables. Las dos formas estables del $\mathrm{Cr}$ son la trivalente (III) y la hexavalente (VI), sin embargo existen otros estados valencia. El Cr VI es el más tóxico pues fácilmente puede oxidarse tiene una capacidad para general radicales libres [1]. El Cr está presente en la naturaleza en concentraciones bajas y es usado en diversos procesos biológicos necesarios para la vida. Tanto el $\mathrm{Cr}$ III como el VI se usan además en procesos industriales, como curtientes, pigmentos y conservantes textiles, catalizadores, agentes anticorrosivos, conservantes de madera, aleaciones, pinturas anti-incrustantes, lodos de perforación, baterías de alta temperatura, fungicidas, recubrimientos metálicos y electrogalvanizados. Una vez expulsado al medio natural el $\mathrm{Cr}$ pasa a formar parte del suelo y el agua, existiendo la posibilidad de ingresar al organismo por vía oral, nasal y tópica [2].

El Cr ingresa al organismo a través de la dieta como $\mathrm{Cr}$ VI y es transformado a Cr III, siendo necesario en pequeñas cantidades para mantener la homeostasis. Este es eliminado generalmente por la orina en 7 días, sin embargo, una parte puede permanecer en las células por varios años convirtiéndose en el origen de diversas afecciones, lo que incluye reacciones alérgicas, erupciones cutáneas, irritación y sangrado de la nariz. Asimismo, la presencia de Cr VI también ha sido asociado a úlceras intestinales y anemia [1, 2]. El Undécimo Informe sobre Sustancias Carcinogénicas del Programa Nacional de Toxicología y otras organizaciones de salud internacionales consideran además que el $\mathrm{Cr} \mathrm{VI}$ es un carcinógeno. Su presencia se ha asociando a cáncer del pulmón y senos paranasales, metalosis e hipotiroidismo subclínico, este último especialmente debido a prótesis de cadera que han liberado este metal [3-9].

Siendo la contaminación por $\mathrm{Cr}$ en agua un hecho alarmante debido a sus efectos deletéreos sobre la salud es importante conocer la normativa nacional e internacional sobre los niveles aceptables de este metal en aguas de consumo y riego. Según criterio de la OMS, los niveles admisibles de $\mathrm{Cr}$ total en agua para el consumo humano deben ser menores de $2 \mu \mathrm{g} / \mathrm{l}$ y su detección por espectrometría de absorción atómica (AAS) 
debe ser de 0,05 a 0,2 $\mu \mathrm{g} / \mathrm{l}$ [10]. Tanto el Texto Unificado de Legislación Secundaria de Medio Ambiente (TULSMA) como el Instituto Nacional Ecuatoriano de Normalización 1108 (INEN 1108) confirman que el límite permisible es de 0,05 mg/L [11, 12]. Asimismo, la Ley 16/2002 regula el proceso de eliminación y tratado de los residuos industriales, lo que incluye $\mathrm{Cr}$.

Guano es un cantón de la provincia de Chimborazo conocido por su actividad industrial que está especializado en la producción de materiales con cuero. Guano por otro lado está ubicado sobre un acuífero y atravesado por el río Guano. El agua del río provee de agua para el riego de cultivos, mientras que el acuífero es la principal fuente de agua de consumo de la comunidad. Por la importancia que tiene el agua para la vida y salud de la comunidad nace la pregunta ¿cumple el agua de Guano con los estándares establecidos respecto a los límites de contaminación por Cr? La presente investigación se realizó con el objetivo de evaluar la cantidad de $\mathrm{Cr}$ VI existente en aguas superficiales de la cuenca del río Guano y en pequeños ojos de agua provenientes del acuífero.

\section{Materiales y Métodos}

Se trata de un estudio observacional que se hizo con el objetivo de comparar los niveles de $\mathrm{Cr}$ VI en el río Guano y ojos de agua que funcionan como afluentes. El río Guano tiene una extensión de $30.05 \mathrm{~km} 2$, tiene dos ojos de agua, como se puede observar en la Figuras 1-3.

Para determinar la cantidad de Cr VI en el río Guano se recolectó muestras de agua a una distancia de $200 \mathrm{~m}$ río abajo, con respecto a la curtiembre más cercana del río (Figura 4). Además, se tomó muestras de los dos ojos de agua del acuífero. Los niveles de Cr VI se determinaron mediante espectrofotometría de Hach. El volumen de agua recolectado para cada muestra fue de aproximadamente $1 \mathrm{~L}$, lo que se hizo en recipientes plásticos completamente estériles.

Para el procesamiento de los datos se usó hojas de cálculo y para el análisis estadístico $\mathrm{R}$ versión 3.6.2. Se calculó medidas de tendencia central como la media y de dispersión como la desviación estándar. Para determinar si existió diferencia estadísticamente significativa entre lo encontrado en las muestras de agua y los valores establecidos como normales de la OMS, TULSMA e INEN 1108 se utilizó la prueba Z.

Para la recolección de muestras de agua se contó con la autorización y ayuda de la Dirección de Aguas del cantón Guano. Dicha institución colaboró además con la información cartográfica empleada para la investigación (Figuras 1-3). 


\section{Resultados y Discusión}

El objetivo del presente estudio fue evaluar los niveles de $\mathrm{Cr}$ VI en aguas del río Guano y acuíferos, los valores obtenidos fueron comparados con los parámetros aceptados por la OMS, TULSMA e INEN 1108 como normales (Tabla 1). Según se puede observar en la Tabla 1, la media en mg/dl de Cr VI encontrada en el río Guano y ojos de agua del acuífero es más alta que los niveles recomendados por la OMS, aunque similares a los establecidos como normales por TULSMA e INEN 1108. Las pruebas de significación estadística sugieren que la media de $\mathrm{Cr}$ del río Guano y ojos de agua es mayor que los parámetros recomendados por la OMS.

Son ampliamente conocidos los efectos del $\mathrm{Cr}$ sobre la salud humana, diversas investigaciones dan fe de que este elemento puede ser el precursor de varios malestares [13]. Pero la enfermedad originada va en dependencia del tipo de $\mathrm{Cr}$ que ingrese al organismo $[5,13,14]$. Aunque el $\mathrm{Cr}$ III es un nutriente esencial para los humanos y la falta de éste puede causar diferentes patologías, no sucede lo mismo con el $\mathrm{Cr}$ VI (hexavalente), el cual en niveles superiores a los permisibles pueden afectar a la salud humana y se convertirse en un factor de riesgo predisponente para el desarrollo de diversas dolencias, tanto sistémicas (diabetes mellitus, alergias, cáncer, etc.) como localizadas, donde resaltan como órganos más afectados la piel expuesta y las mucosas $[5,15]$. La vía de ingreso del $\mathrm{Cr}$ VI a nuestro organismo es variable puede ser ingerido a través del agua de consumo o alimentos contaminados, o a través de la respiración [5].

Conociendo que en el cantón Guano el 15\% de su población se dedica a la manufactura del cuero, actividad económica que aumenta significativamente la exposición a dicho metal, así como el riesgo de contaminación del medio ambiente, Ilama la atención el hecho de que los niveles de $\mathrm{Cr}$ encontrados en el agua del río y ojos de agua se encuentran dentro de los parámetros aceptados por las normas TULSMA e INEN 1108, pero resultan ser superiores a los que establece la OMS. Por lo cual se debe constituir un punto de partida para desarrollar un trabajo encaminado a regir un control sanitario respecto a los niveles de $\mathrm{Cr}$ como de otros químicos, con el objeto de evitar los efectos nocivos que puedan provocar sobre la salud humana [16-18].

Cabe recalcar que, debido a la cantidad de muestras tomadas para este estudio por factores económicos, sus resultados podrían estar influenciados por el error tipo $\beta$. Pero existen varias investigaciones las cuales corroboran con que el $\mathrm{Cr}$ se halla elevado en zonas cercanas a las industrias donde utilizan este elemento químico. Así en una investigación realizada en Argentina en el ambiente de una curtiembre se obtuvieron concentraciones de $\mathrm{Cr}$ hexavalente similares a las encontrados en la presente investigación dentro de aguas subterráneas y superficiales [12], lo cual confirma el riesgo de contaminación del agua en sectores cercanos a procesamiento de pieles. De forma 
similar un artículo publicado en el 2011 analiza el riesgo que representa la exposición al Cr VI en el mecanismo de producción del cáncer [19]. Una investigación realizada en Grecia en el 2014, sin tener en cuenta un ambiente de curtiembre arrojó cifras de $\mathrm{Cr}$ hexavalente dentro de límites normales; del análisis de estas investigaciones se desglosa que existe una relación indiscutible entre el riesgo de contaminación de las aguas y la cercanía de las industrias que utilizan este elemento químico [20]. Por otro lado, también se evidencian intoxicaciones por $\mathrm{Cr}$, en personas que se han sometido a artroplastia de revestimiento cadera, demostrando una vez más la toxicidad de este material en dosis elevadas [21].

En América Latina las curtiembres causan contaminación especialmente en el agua dando dificultades ambientales, intoxicando la biodiversidad por la descarga de altas concentraciones de material inorgánico especialmente cromo hexavalente y la realidad ecuatoriana de algunas ciudades donde el sustento económico prima, ante todo, las curtiembres no presentan planes de cuidado y protección ambiental en cumplimiento de la normativa vigente para crear un compromiso social y responsable [22]. Por otra parte, es importante incluir medidas de recuperación del cromo con enfoque preventivo basándose en programas de producción más limpia (PML) en el sector de las curtiembres [23]. La estrategia de PML es un punto de encuentro entre la productividad y el manejo ambiental en la que se aplica en concepto de prevención de la contaminación y eficiencia energética mediante la implementación de las tres R's (Reciclaje, Reúso, Recuperación) tratamiento y disposición final de desechos [24].

La implementación del programa PML en otros países como Colombia están desde año 2002, lo que les ha permitido mejorar los procesos productivos, entre ellos la recuperación de cromo con varios métodos como los químicos con precipitación con $\mathrm{MgO}$ y re dilución con $\mathrm{H} 2 \mathrm{SO}$, con eficiencias de 90\% [25]. Los métodos biológicos pueden ser usados, tanto para el tratamiento de los desechos al final del proceso como para la recuperación y reúso de los efluentes del cromo, donde la biorremediación requiere microorganismos, principalmente bacterias y hongos que destruyen o transforman materiales peligrosos [26], estos procesos ofrecen alta especificidad mediante mecanismos de bioadsorción, bioacumulación, biotransformación o biolixiviación son económicos, seguros que las tecnología convencionales [27].

Se han reportado un buen número de estudios con la aplicación potencial de microorganismos como agentes biolixiviadores (removedores de metales) se puede promover la reducción de $\mathrm{Cr}+6$, los procesos enzimáticos claramente involucrados contribuyen a inmovilizar el metal [28]. Por otro lado, Zahor and Rehman, encontraron que Bacillos sp. y Staphylococcus capitis redujeron de cromo hexavalente a trivalente con una eficacia del $86 \%$ y $89 \%$ respectivamente, después de 144 hr de exposición a efluentes industriales $[29,30]$. En otro estudio con Bacillus sp. se obtuvo la capacidad de tolerar el cromato y reducirlo mediante la actividad de una reductasa, mezclada 
con un mecanismo de expulsión de cromo, estas peculiaridades la hacen muy útil en biorremediación de ambientes contaminados con cromo [31]. En otra investigación se experimentan formas para separar el cromo hexavalente con fitorremediación usando vegetales, hongos y algas, estudios que por el proceso en la mayoría de los casos el inconveniente es el tiempo, pero aun así es una buena manera de usar recursos disponibles en la naturaleza [32].

Dentro de la investigación se recomienda a futuro la recolección de muestras representativas incluyendo datos como el caudal, $\mathrm{pH}$ - temperatura de agua y ambiente para completar la investigación; así como considerar la efectividad de la prueba empleada se puede optar por otras técnicas como el espectrofotómetro ultravioleta por su mejor especificidad y precisión en los resultados, por otra parte es importante incluir medidas de recuperación del $\mathrm{Cr}$ con enfoque preventivo basándose en programas de producción más limpia en el sector de las curtiembres.

\section{Conclusiones}

Nuestros hallazgos sugieren que los niveles de $\mathrm{Cr}$ en el agua de riego y de consumo de esta región se encuentran dentro del rango permitido por las normas vigentes en Ecuador. Sin embargo, al comparar estos resultados con los recomendados a nivel internacional por la OMS, se encuentran muy por encima de ellos. Por este motivo se recomienda se tomen medidas adecuadas para reducir la contaminación del agua por $\mathrm{Cr}$ y de este modo evitar además la filtración de $\mathrm{Cr}$ al acuífero sobre el que reposa Guano. Se considera además fundamental iniciar un control riguroso de metales pesados en el agua.

\section{Contribución de los Autores}

Las distintas fases de investigación fueron realizadas por todos los autores, quienes contribuyeron de igual forma en todo el proceso. El autor correspondiente representa al colectivo de autores.

\section{Financiamiento}

Los recursos fueron provistos de los autores. 


\section{Disponibilidad de Datos}

Los datos que sustenta este manuscrito se encuentran disponibles bajo solicitud al autor de correspondencia.

\section{Agradecimientos}

Nuestro sincero agradecimiento a Liliana Teruel, Emily Morillo, Pablo Estrella y Karla Arias por su ayuda para la realización de la investigación. De la misma manera agradecemos al Municipio de Guano por el apoyo y facilitación de los mapas satelitales necesarios para el presente.

\section{Conflicto de Intereses}

No existe conflicto de interés alguno.

\section{References}

[1] Shanker A, Venkateswarlu B. Chromium: Environmental pollution, health effects and mode of action. Encyclopedia of Environmental Health. 2011;1:624-633.

[2] United States Department of Labor. [Página principal en internet], Washington, DC: OSHA; 2018. Disponible en: www.osha.gov/SLTC/hexavalentchromium

[3] California Office of Environmental Health Hazard Assessment. [Página principal en internet]. California: OEHHA; 2016. Disponible en: https://oehha.ca.gov/media/ downloads/faqs/sphexchromiumairfact111616.pdf

[4] González A, Colín L, Bizarro P et al. Pollution by metals: Is there a relationship in glycemic control?. J. Environmental Toxicology and Pharmacology. 2016;46:337-343.

[5] Tseng C-H, Lee $\mathrm{I}-\mathrm{H}$, Chen Y-C. Evaluation of hexavalent chromium concentration in water and its health risk with a system dynamics model. Sci Total Environ. 2019;669:103-111.

[6] Instituto Nacional Del Cáncer. USA: NIH; 2015. Disponible en: https://www.cancer. gov/espanol/cancer/causas-prevencion/riesgo/sustancias/Cr

[7] Hernández F, Conde L. Contaminantes químicos. Conceptos básicos en toxicología industrial. Criterios de toxicidad. Protección individual frente a contaminantes químicos. Tratado de medicina del trabajo. $3^{\text {rd }}$ ed. Spain: Elsevier; 2019.

[8] Bayhana T, Ünal S, Cırak E et al. Heavy metal levels in patients with ineffective erythropoiesis. J. Transfusion and Apheresis Science. 2017;56(4):539-543. 
[9] Lenntech BV. Propiedades químicas del $\mathrm{Cr}$ - Efectos del $\mathrm{Cr}$ sobre la Salud Efectos ambientales del Cr. 2019 Disponible en: https://www.lenntech.es/periodica/ elementos/cr.htm

[10] Instituto Ecuatoriano de Normalización. Quito: INEN;2014. Disponible en: http://www. pudeleco.com/files/a16057d.pdf

[11] Matteoda E, Blarasin M, Damilano G, Cabrera A. Cr en aguas subterráneas y superficiales en el entorno de una curtiembre, relación con valores de fondo natural. Córdoba: Elena;2009.

[12] Mendoza B, Tipán N. Estudio hidráulico del río guano para la determinación de la conductancia entre río- acuífero y zonas de inundación. 2018. Disponible en: http://dspace.unach.edu.ec/handle/51000/4983.

[13] Gónzalez X, Alonso J, Tadín N, Santaliestra A. Tóxicos específicos- Clinicalkey. 67:351-559.

[14] Becerra S, Soria C, Jaramillo F, Moreno J. Trastornos a la salud inducidos por $\mathrm{Cr}$ y el uso de antioxidantes en su prevención o tratamiento. J. of Pharmacy \& Pharmacognosy Research. 2014;2(2):19-30.

[15] Achmad R, Budiawan, Auerkari E. Effects of chromium on human body. Annu Res Rev Biol. 2017;13(2):1-8, 2017.

[16] Organización Mundial de la Salud. Catalogación por la Biblioteca de la OMS. Guía para la Calidad del Agua Potable. Disponible en: https://www.who.int/water_ sanitation_health/dwq/gdwq3_es_fulll_lowsres.pdf

[17] Franchini CA, Mutti A, Cavatorta E, Predoni C, Borghetti A. Indicadores biológicos para la valoración de la exposición humana a compuestos químicos industriales. Disponible en: http://publicaciones.san.gva.es/publicaciones/documentos/V. 1851-1993.PDF

[18] AENOR. Presidencia De La Republica. Norma de calidad ambiental y de descarga de efluentes: Recurso agua. Disponible en: http://extwprlegs1.fao.org/docs/pdf/ ecu112180.pdf

[19] Rakhunde R, Deshpande L, Juneja HD. Chemical speciation of chromium in water: A review. Crit Rev Environ Sci Technol. 2012;42(7):776-810.

[20] Fontaine M, Clement Y, Blanc N, Demesmay C. Hexavalent chromium release from leather over time natural ageing vs accelerated ageing according to a multivariate approach. J Hazard Mater. 2019;368:811-8.

[21] Czekaj J, Ehlinger M, Rahme M, Bonnomet F. Metallosis and cobalt - chrome intoxication after hip resurfacing arthroplasty. Journal of Orthopaedic Science. 2016;21(3):389-394.

[22] Guajala MER, Abril CMM, Falcón LMM, Chalán PAL. Gestión económica ambiental del sector curtiembre de Ambato. Augusto Guzzo Revista Acadêmica. 2016;17:133-142. 
[23] Benitez-Campo N. Producción limpia y biorremediación para disminución de la contaminación por cromo en la industria de curtiembres. Ambiente y sostenibilidad. 2011;1:25-31.

[24] Restrepo I, Vásquez PA, Valderrutén C, Jaramillo AD, Vivas GA. Cadena del cuero en el Valle del Cauca: PML para el mejoramiento de la competitividad. Colombia; 2006. 107 p.

[25] D’Agostino M, Cook N, Di Bartolo I et al. Section 3.1 Detection Methodology. Monitoring, identification, genotyping and inactivation of enteric viruses in the food chain. 5, 85.

[26] Maier RM, Pepper IL, Gerba CP. Environmental Microbiology. $2^{\text {nd }}$ edition. California: Elsevier; 2009

[27] Vásque Ñz C, Paola A, Rengifo Valderrutén C, Salazar Jaramillo DA. Cadena del cuero en el Valle del Cauca: PML para el mejoramiento de la competitividad. 2006.

[28] Cheung KH, Gu JD. Reducción de cromato (CrO42-) por un consorcio de enriquecimiento y un aislado de bacterias marinas reductoras de sulfato. Chemosphere. 2003;52(9);1523-1529.

[29] Zahoor A, Rehman A. Aislamiento de bacterias reductoras de $\mathrm{Cr}$ (VI) a partir de efluentes industriales y su uso potencial en la biorremediación de aguas residuales que contienen cromo. Revista de Ciencias Ambientales. 2009;21(6):814- 820.

[30] Yang J, He M, Wang G. Eliminación de cromato tóxico utilizando células bacterianas reductoras de $\mathrm{Cr}(\mathrm{VI})$ libres e inmovilizadas de Intrasporangium sp. Q5-1. World Journal of Microbiology and Biotechnology. 2009;25(9):1579-1587.

[31] Elangovan R, Abhipsa S, Rohit B, Ligy P, Chandraraj K. Reduction of $\mathrm{Cr}$ (VI) by a Bacillus sp. Biotechnology Letters. 2006;28(4):247-252.

[32] Quezada R, Varela E, Rosa MA. Remediación natural para completar la depuración del cromo (VI) en efluentes de curtiembres. Quinto Congreso Deficiencia y Tecnología para Alumnos, Simposio Llevado a cabo en el Congreso de la Facultad Regional de Villa María, Córdoba, Argentina. (pp. 15-16. 2012. 